\title{
Clinical Failure of Vancomycin Treatment of Staphylococcus aureus Infection in a Tertiary Care Hospital in Southern Brazil
}

\author{
Larissa Lutz, Adão Machado, Nadia Kuplich \\ and Afonso Luís Barth
}

\begin{abstract}
Biomedical research Unit, Microbiology Unit, Clinical Pathology Service - Clinical Hospital of Porto Alegre, RS, Brazil
\end{abstract}

\begin{abstract}
We describe a case of clinical failure of vancomycin treatment of Staphylococcus aureus infection and the laboratory characteristics of the organism in a tertiary referral university hospital in southern Brazil. An 11-month-old male patient presented with pneumonia and $S$. aureus was isolated from his respiratory tract. Initial treatment with oxacillin and gentamicin was ineffective. Vancomycin was added to the regimen as the patient worsened, but after the $30^{\text {th }}$ day of vancomycin treatment $S$. aureus was isolated from the blood. This isolate had a minimum inhibitory concentration (MIC) for vancomycin of $4 \mu \mathrm{g} / \mathrm{mL}$. After pre-incubation with vancomycin the isolate displayed an increase in the expression of vancomycin resistance and colonies grew in the presence of up to $12 \mu \mathrm{g} / \mathrm{mL}$ vancomycin. Based on these results, and considering that the patient had not responded to vancomycin, the isolate was considered to be $S$. aureus heteroresistant to vancomycin (SAHV). The SAHV proved to be similar, based on DNA macrorestriction analysis, to methicillin resistant $S$. aureus (MRSA) isolates from other patients in the hospital who had responded to vancomycin treatment. Our findings underline the need to improve methods in the clinical laboratory to detect the emergence of $S$. aureus clinically resistant to vancomycin. The fact that the isolate emerged in the blood 30 days after vancomycin treatment was initiated suggests that the organism was originally an MRSA that had acquired the ability to circumvent the mechanism of action of vancomycin.
\end{abstract}

Key Words: S. aureus, resistance, vancomycin.

Staphylococcus aureus is one of the most common causes of nosocomial and community-acquired infection. Since the emergence of methicillin resistant S. aureus (MRSA), the glycopeptide vancomycin has been the only uniformly effective treatment of staphylococcal infections [1]. In May 1996, Hiramatsu et al. [2] described the first clinical isolate of S. aureus with reduced susceptibility to vancomycin. One year later, two similar organisms were reported in the United

Received on 29 August 2002; revised 16 January 2003.

Address for correspondence: Dr.Afonso Luís Barth. Hospital de Clínicas de Porto Alegre. Unidade de Pesquisa Biomédica - Serviço de Patologia Clínica. Rua Ramiro Barcelos 2350. Porto Alegre, RS, Brazil, Zip code: 90.035-003. Phone: (+51) 33168607 Fax:+5133168310. E-mail: albarth@hcpa.ufrgs.br

The Brazilian Journal of Infectious Diseases 2003;7(3):224-228 (C) 2003 by The Brazilian Journal of Infectious Diseases and Contexto Publishing. All rights reserved.
States [3]. The main feature of these isolates was that they exhibited decreased susceptibility to vancomycin in vivo, after prolonged exposure to the drug [2]. The minimal inhibitory concentration (MIC) of vancomycin was $8 \mu \mathrm{g} / \mathrm{mL}$ for these three isolates, however other $S$. aureus isolates with lower MIC for vancomycin $(4 \mu \mathrm{g} /$ $\mathrm{mL}$ ) may also be resistant in vivo. The latter are termed S. aureus heteroresistant to vancomycin, and they account for up to $20 \%$ of the MRSA isolates from Japanese university hospitals [4]. Isolates of S. aureus with reduced susceptibility to vancomycin have also been described elsewhere, including Brazil [5-8]. The presence of such organisms may explain the failure of vancomycin to treat some MRSA infections.

We examined a case of clinical failure of vancomycin treatment of $S$. aureus infection in a tertiary referral university hospital in southern Brazil and we investigated the laboratory characteristics of this bacteria. 


\section{Materials and Methods}

Bacteriological cultures were prepared according to conventional diagnostic methods. Briefly, blood cultures were made with the Bactec $\AA$ system (Becton Dikinson, Sparks, USA) and the appearance of colonies after subculture to brain-heart infusion agar containing 5\% sheep blood - BAP (Oxoid, Basingstoke, Hampshire, UK) was used to screen for staphylococci. Staphylococcus aureus was confirmed by the coagulase test. Isolates were tested for susceptibility to penicillin $(10 \mu \mathrm{g})$, oxacillin $(1 \mu \mathrm{g})$, amoxicillin/clavulanic acid (20/ $10 \mu \mathrm{g})$, erythromycin $(15 \mu \mathrm{g})$, gentamicin $(10 \mu \mathrm{g})$, clindamycin $(2 \mu \mathrm{g})$, rifampin $(5 \mu \mathrm{g})$, trimethoprimsulfamethoxazole $(1.25 / 23.75 \mu \mathrm{g})$ and vancomycin $(30 \mu \mathrm{g})$ by the disk diffusion method, according to NCCLS [9]. The minimal inhibitory concentration (MIC) of amoxicillin-clavulanate, amikacin and vancomycin was determined by the E-Test (AB Biodisk, Solna, Sweden). A heavy inoculum of $S$. aureus (adjusted to a turbidity equal to McFarland 2.0) was subcultured onto brain heart infusion (BHI) agar (Oxoid, Basingstoke, Hampshire, UK) containing a range of vancomycin (Sigma, St. Louis, Mo) concentrations [10], in order to test the effect of pre-incubation with vancomycin on the expression of vancomycin resistance by the bacteria. The colonies that grew in the presence of vancomycin were inoculated onto blood agar plates (Oxoid, Basingstoke, Hampshire, UK), without vancomycin, and incubated at $35^{\circ} \mathrm{C}$ for $24 \mathrm{~h}$. This procedure was repeated daily for 18 days. At days 3, 6, 12 and 18 of passage, vancomycin MIC was determined by the E-Test. Serial passage was discontinued when the MIC of vancomycin had decreased to the initial MIC [11]. Macrorestriction analysis of chromosomal DNA with SmaI and pulsedfield gel electrophoresis (PFGE) was performed as previously described [12].

\section{Case Report}

An 11-month-old male patient was admitted to the Paediatric Intensive Care Unit at Hospital de Clínicas de Porto Alegre in June 1998. The infant presented with secondary sepsis due to pneumonia at admission.
Staphylococcus aureus was isolated from pleural fluid and oxacillin (200mg/kg/day) and gentamicin ( $75 \mathrm{mg} / \mathrm{kg} /$ day) were given, since the isolate displayed in vitro susceptibility to these antibiotics. The patient developed septicaemia and multiple organ failure despite treatment, and after 10 days $S$. aureus, susceptible only to vancomycin and rifampin, was isolated from a tracheal aspirate. Oxacillin was replaced by vancomycin $(60 \mathrm{mg} /$ $\mathrm{kg} / \mathrm{day}$ ), however rifampin was not used as the patient had significant hepatic dysfunction. After 25 days of vancomycin treatment the patient worsened and $S$. aureus was again isolated from the blood. The dose of vancomycin was increased to achieve a serum concentration with the maximum recommended levels $(10 \mathrm{mg} / \mathrm{L}$ at trough and $40 \mathrm{mg} / \mathrm{L}$ at peak). After a short period of clinical improvement, the patient again worsened and $S$. aureus was isolated from the blood on the $30^{\text {th }}$ day of vancomycin treatment. This isolate remained resistant to oxacillin and susceptible to vancomycin according to the disk diffusion method. The MIC of vancomycin proved to be $3 \mu \mathrm{g} / \mathrm{mL}$ by the E-Test, but was reported as $4 \mu \mathrm{g} / \mathrm{mL}$, according to the recommendation of the manufacturer. The Public Health Laboratory Service (PHLS) in London, UK confirmed that the isolate was an MRSA, based on the presence of the mecA gene and that the MIC for vancomycin was $4 \mu \mathrm{g} / \mathrm{mL}$, by the microdilution method. At this point vancomycin was administered in continuous infusion to obtain constant serum levels of 30 to $40 \mu \mathrm{g} /$ $\mathrm{mL}$. Amikacin was administered in a single daily dose of $20 \mathrm{mg} / \mathrm{kg}$, because at the peak of the infusion, serum levels were $68 \mu \mathrm{g} / \mathrm{mL}$, higher than the MIC for this S. aureus $(62 \mu \mathrm{g} / \mathrm{mL})$. Amoxicillin-clavulanate was administered in four daily doses of $50 \mu \mathrm{g} / \mathrm{mL}$, because the serum level obtained by this mode of administration $(120 \mu \mathrm{g} / \mathrm{mL}$ at the peak) was also higher than the MIC for the organism $(32 \mu \mathrm{g} / \mathrm{mL})$. The patient stabilised and amikacin was withdrawn from the treatment after 21 days. After 107 days of vancomycin treatment, the patient left the hospital with serious pulmonary sequels. He was tracheostomized, and in the ensuing months he had numerous respiratory problems. His left lung, which was strongly damaged, was removed, although the right lung also had significant residual lesions. The patient was re-admitted to the hospital in May 2000 , but he subsequently died of pneumothorax. From 
Figure 1. Colonial morphology of Staphylococcus aureus heteroresistant to vancomycin (SAHV) on brainheart infusion agar containing 5\% sheep blood.

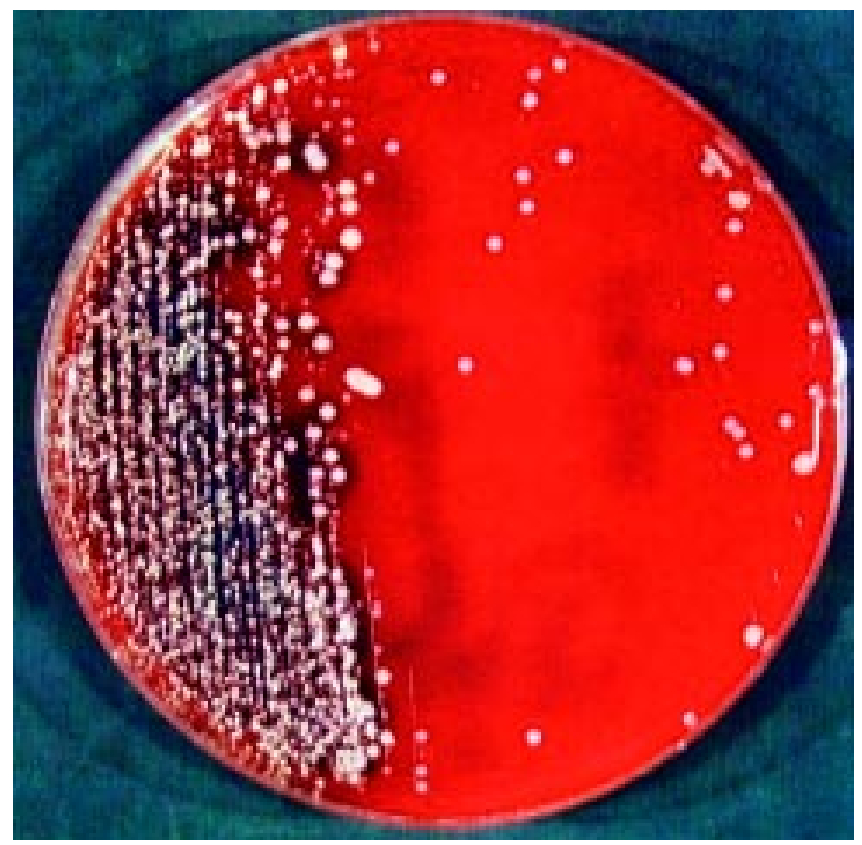

the time of the first admission of the patient to our hospital, no bacterium, other than $S$. aureus, was obtained from the microbiological cultures of the specimens that were collected.

\section{Laboratory Findings}

The $S$. aureus obtained from the blood culture of the patient after 30 days of vancomycin treatment displayed a heterogeneous colonial morphology on BAP (Figure 1). The isolate was considered susceptible to vancomycin, but it was resistant to all other antibiotics according to the disk diffusion method, and the MIC for vancomycin was $4 \mu \mathrm{g} / \mathrm{mL}$. After pre-incubation with vancomycin the isolate increased the expression of vancomycin resistance and the colonies grew in the presence of up to $12 \mu \mathrm{g} / \mathrm{mL}$ of vancomycin (Figure 2). The colonies were then inoculated onto blood agar plates without vancomycin and incubated for 18 days; at days 3, 6, 12 and 18 after passage the vancomycin MIC by the E-Test was $12,8,8$ and $2 \mu \mathrm{g} / \mathrm{mL}$, respectively. Based on these results the isolate was considered to $S$. aureus heteroresistant to vancomycin (SAHV).
The PFGE pattern of SmaI - digested genomic DNA of this SAHV was compared with DNA digests of MRSA from three other patients hospitalised during the same period, who had responded satisfactorily to vancomycin treatment. The DNA patterns were very similar (Figure 3); the SAHV differed from the three MRSA by less than six bands [13].

\section{Discussion}

We have described the characteristics of a $S$. aureus isolate obtained from a patient who did not respond to vancomycin treatment. It has been suggested that therapeutic failure of vancomycin treatment is associated with low level resistance, or heteroresistance, to vancomycin [6]. The S. aureus described here displayed a borderline MIC for vancomycin and was considered susceptible to vancomycin by the disk diffusion method. This is a case for concern [14]. The routine susceptibility tests did not correlate with the clinical resistance of the isolate. Neither the disk diffusion nor the MIC determination proved to be good parameters for the detection of $S$. aureus with 
Figure 2. Minimum inhibitory concentration of vancomycin $(\mu \mathrm{g} / \mathrm{mL})$ for Staphylococcus aureus heteroresistant to vancomycin (SAHV) after pre-incubation with vancomycin.

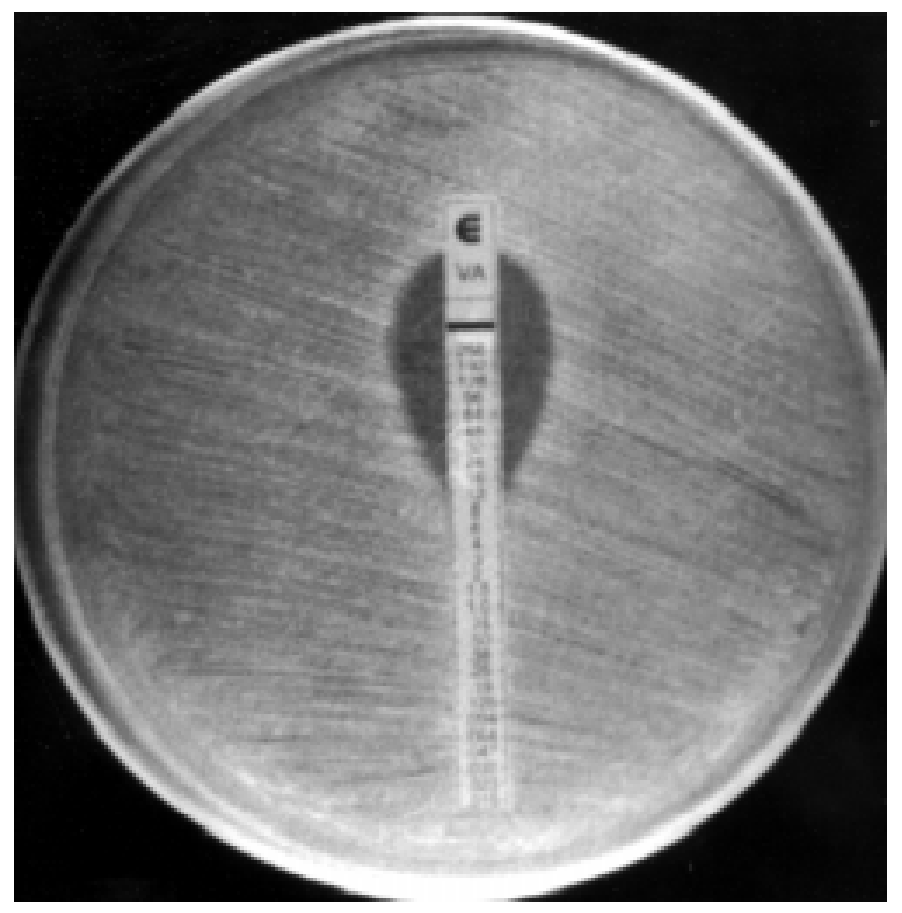

Figure 3. Macrorestriction profile of Staphylococcus aureus heteroresistant to vancomycin - SAHV (D) and three methicillin resistant S. aureus - MRSA (A, B and C) isolates from inpatients at the Hospital de Clínicas de Porto Alegre. MWM = Molecular Weight Marker (lambda ladder of 48.5Kb).

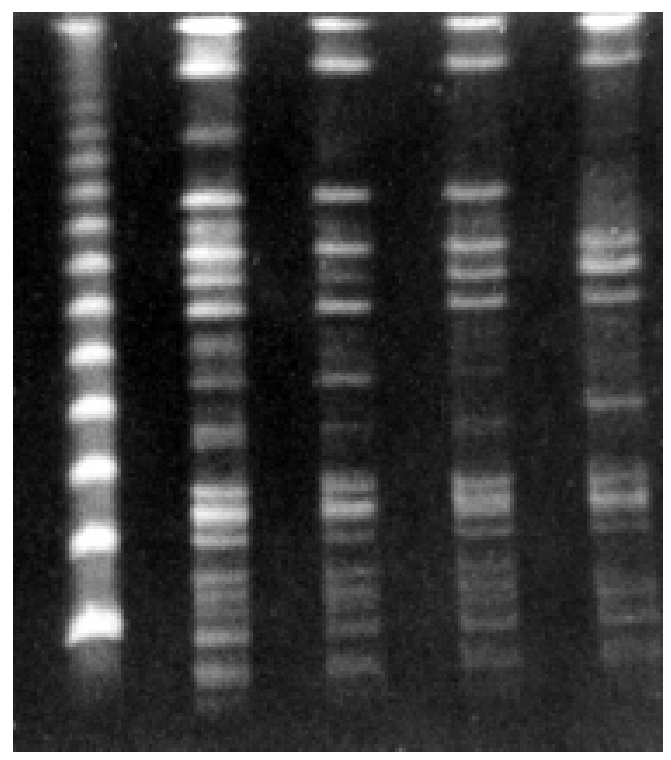


reduced susceptibility to vancomycin. This suggests that it is necessary to improve clinical methodology to detect the emergence of $S$. aureus clinically resistant to vancomycin. We were able to increase vancomycin resistance in our isolate by passing it through increasing concentrations of this antibiotic. After serial passages in media with vancomycin the $S$. aureus was able to grow in the presence of up to $12 \mu \mathrm{g} / \mathrm{mL}$ of this antibiotic; the MIC reverted to a level lower than the original (4 $\mu \mathrm{g} / \mathrm{mL}$ ), after multiple passages of the isolate in media free of vancomycin. This profile is compatible with that of isolates that have been classified as heteroresistant [11]. This method may not be specific for the detection of heteroresistant $S$. aureus and has to be used with caution for isolates that are not involved with clinical failure of vancomycin therapy [15]. However, if the phenomenon of vancomycin resistance after preincubation also occurs in vivo it would explain the therapeutic failure of vancomycin treatment in this case.

On the other hand, the SAHV described in this study had very similar DNA patterns to other MRSA from our hospital that were clinically susceptible to vancomycin. This finding, taken together with the fact that the $S$. aureus was obtained from the patient's blood after 30 days of vancomycin treatment, indicates that the bacterium was originally an MRSA that acquired the ability to circumvent the mechanism of action of vancomycin.

\section{Acknowledgments}

We are indebted to Dr. Tyrone L. Pitt for critical review of the manuscript. We also thank the Central Public Health Laboratory, Colindale, UK, for confirming the MIC of vancomycin and PCR detection of the mecA gene in the isolate.

\section{References}

1. Pfaller M.A., Jones R.N., Doern G.V., et al. The SENTRY Participants Group. Survey of blood stream infections attributable to Gram-positive cocci: Frequency of occurrence and antimicrobial susceptibility of isolates collected in 1997 in the United States, Canada, and Latin American from the SENTRY Antimicrobial Surveillance Program. Diagn Microbiol Infect Dis 1999;33:283-97.
2. Hiramatsu K., Hanaki H., Ino T., et al. Methicillin-resistance Staphylococcus aureus clinical strain with reduced vancomycin susceptibility. J Antimicrob Chemother 1997;40:135-46.

3. Centers for Disease Control and Prevention. Staphylococcus aureus with reduced susceptibility to vancomycin - United States, 1997. Morbid Mortal Weekly Rep 1997;46:765-6.

4. Hiramatsu K., Aritaka N., Hanaki H., et al. Dissemination in Japanese hospitals of strains of Staphylococcus aureus heterogeneously resistant to vancomycin. Lancet 1997;350:1670-3.

5. Turco T.F., Melko G.P., Williams J.R. Vancomycin intermediate-resistant Staphylococcus aureus. Ann Pharmacother 1998; 32: 758-60.

6. Ploy M.C., Grelaud C., Martin C., et al. First clinical isolate of vancomycin-intermediate Staphylococcus aureus in a French hospital. Lancet 1998;351:1212.

7. Rotun S.S., McMath V., Schoonmaker D.J., et al. Staphylococcus aureus with reduced susceptibility to vancomycin isolated from a patient with fatal bacteremia. Emerg Infect Dis 1999;5:147-9.

8. Oliveira G.A., Dell'Aquila A.M., Masiero R.L., et al. Isolation in Brazil of nosocomial Staphylococcus aureus with reduced susceptibility to vancomycin. Infect Control Hosp Epidemiol 2001;22:443-8.

9. NCCLS. Performance standards for antimicrobial susceptibility testing. Eighth Informational Supplement M100-S8. National Committee for Clinical Laboratory Standards, Villanova, PA, 1998.

10. Howe R.A., Wootton M., Walsh T.R., et al. Expression and detection of hetero-vancomycin resistance in Staphylococcus aureus. J Antimicrob Chemother 1999;44:675-8.

11. Boyle-Vavra S., Berke S.K., Lee J.C., Daum R.S. Reversion of the Glycopeptide Resistance Phenotype in Staphylococcus aureus Clinical Isolates. Antimicrob Agents Chemother 2000;44:272-7.

12. Kaufmann M.E., Pitt T.L. Pulsed-field gel electrophoresis of bacterial DNA. In: Chart H., ed. Methods in Practical Laboratory Bacteriology. London: CRC Press. 1994.

13. Tenover F.C., Arbeit R.D., Goering R.V., et al. Interpreting chromosomal DNA restriction patterns produced by pulsed-field gel electrophoresis: criteria for bacterial strain typing. J Clin Microbiol 1995;33:2233-9.

14. Tenover F.C., Lancaster M.V., Hill B.C., et al. Characterization of Staphylococci with Reduced Susceptibilities to Vancomycin and Other Glycopeptides. J Clin Microbiol 1998;36:1020-7.

15. Aucken H.M., Warner M., Ganner M., et al. Twenty months of screening for glycopeptide intermediate Staphylococcus aureus. J Antimicrob Chemother 2000;46:639-40. 